(C) <2021>. This manuscript version is made available under the CC-BY-NC-ND 4.0 license http://creativecommons.org/licenses/by-nc-nd/4.0/

The definitive publisher version is available online at https://doi.org/ 10.1016/j.sapharm.2021.04.013 


\section{A participatory research approach in 2 community pharmacy research: the case 3 for video-reflexive ethnography \\ 4 Authors}

5 Faith R. Yong, Doctoral Candidate. ${ }^{\text {F }}$ Faith.Yong@uts.edu.au

6 Dr Su-Yin Hor, Lecturer. ${ }^{\text {b Suyin.Hor@uts.edu.au }}$

7 Professor Beata V. Bajorek. ${ }^{\text {B Beata.Bajorek@uts.edu.au }}$

8 Corresponding Author: Faith R. Yong

9 Affiliation

10 a Discipline of Pharmacy, Graduate School of Health, University of Technology Sydney. 100

11 Broadway, Chippendale, Sydney NSW 2008, Australia.

$12{ }^{b}$ Centre for Health Services Management, Faculty of Health, University of Technology Sydney.

13 Building 10, 15 Broadway, Ultimo, Sydney NSW 2007, Australia.

\section{ABSTRACT}

Video-reflexive ethnography (VRE) is a qualitative methodology that explores the complex nature of healthcare 'as it really is'. Its collaborative and reflexive process invites stakeholders (e.g. pharmacists and pharmacy support staff) to participate in analysing their everyday work practices as captured on video footage. Through close collaboration with practitioners and attention to their work contexts, VRE may be a useful methodology to engage a time-poor pharmacy workforce in research about themselves, encouraging more practitioner involvement in practice-based research. 
change in healthcare settings, and could be effective in provoking change in otherwise resistant pharmacy environments.

Much like traditional ethnographic approaches, VRE researchers have relied on being present 'in the field' to observe, record and make sense of practices with participants. The COVID-19 pandemic however, has introduced restrictions around travel and physical distancing, which has required researchers to contemplate the conduct of VRE 'at a distance', and to imagine new ways in which the methodological 'closeness' to stakeholders and their workplace contexts can be maintained when researchers cannot be on site.

In this commentary, we outline the rationale for participatory methods, in the form of VRE, in pharmacy research. We describe the underlying principles of this innovative methodology, and offer examples of how VRE can be used in pharmacy research. Finally, we offer a reflexive account of how we have adapted the method for use in community pharmacy research, to adapt to physical distancing, without sacrificing its methodological principles. This paper offers not only a new methodology to examine the complexity of pharmacy work, but demonstrates also the responsiveness of VRE itself to complexity, and the potential breadth of future research applications in pharmacy both during and beyond the current pandemic.

Keywords: video-reflexive ethnography, community pharmacy, stakeholders, methodology

\section{Introduction}

The work of community pharmacists across the world is significantly impacted by pharmacy practice research. As in USA, ${ }^{1}$ Australia, ${ }^{2}$ and many other countries, pharmacy practice research into cognitive pharmacy services have demonstrated their economic benefits, ${ }^{3-8}$ resulting in their incorporation into public health programs. For example, in Australia, the work of academia, pharmacy associations and government bodies to implement these initiatives have resulted in significant changes in community pharmacy practice. ${ }^{9-12}$ However, few studies have investigated the 
direct effects of these changes on the pharmacy workforce. In the UK, research into the effects of a new federal contract funding administrative and additional services showed an increase in mental and quantitative workload, and increased strain and turnover in community pharmacists. ${ }^{13-16} \mathrm{It}$ is likely the pharmacist workforce internationally are experiencing similar challenges with the fast rate of change. ${ }^{17}$.

The active engagement of front-line pharmacist voices in certain aspects of the research process (e.g. the formulation of research questions, data analysis, interpretation and translation of findings into practice) is necessary in order for pharmacy practice research to remain directly translatable to the profession. Allowing frontline clinicians to analyse, discuss and potentially translate findings back to their everyday work settings could be transformational in impact. While clinician participation in content validation appears to be the norm in qualitative pharmacy research, the lack of frontline clinician involvement in other research processes (e.g. co-analysis and formulating research questions) could limit the value and impact of research findings into the pharmacy setting.

It should be noted at this point that active engagement of pharmacists in research has not been an easy task, historically. Various research around the world has shown that pharmacists do not frequently engage with research teams, citing workload and time constraints despite recognising its value. $^{11,18-23}$ Other factors such as lack of training or post-graduate studies are known to affect pharmacists carrying out research. ${ }^{20-23}$ We discuss some other possible reasons below. It has been acknowledged that new approaches to community pharmacy research are necessary: complex social phenomena, behaviours and beliefs are not well suited to quantitative approaches. ${ }^{24,}$ ${ }^{25}$ The value of qualitative study methods in eliciting such information could be indispensable ${ }^{26}$ in better documenting the contrary behaviour displayed by pharmacists towards providing new health services and engaging in research. 
As an example, a poor understanding of pharmacist work and outdated workload measurements

71 (which were often gathered using quantitative data collection ${ }^{27}$ ) may be a cause of current workforce issues. ${ }^{16,28-33}$ This may be because community pharmacist work is more complex than previously recognised. ${ }^{16,30,34}$ Many processes are enacted by different pharmacy team members and may occur in swift or parallel succession, under the watchful eye of the pharmacist and general public whom they directly interact with. ${ }^{35-41}$ Such processes must be multi-tasked whilst also maintaining a watchful eye on the running of the pharmacy. ${ }^{35,37,42}$ Additionally, the role of the community pharmacist is commonly misperceived by other healthcare professionals and the public alike, who may see them as little more than glorified medication shopkeepers. ${ }^{43-47}$ Lastly, new intervention protocols tend to be clinically focused and do not always consider how services may be perceived and integrated into existing workflows for front-line community pharmacists, who must supervise the pharmacy on top of their clinical duties. ${ }^{35,37,42}$ This has perhaps resulted in a pharmacist workforce that may be wary of outsider influences, including external researchers. ${ }^{11,18,19}$

It has been said that pharmacists are more likely to engage in research where they have a personal interest in the topic of research, and the belief that pharmacy practice research is beneficial, especially to their patients. ${ }^{11,18,19}$ Participatory forms of research, therefore, may be attractive to this population. In particular, participatory approaches that engage with frontline practitioner knowledge and the complexity of their work. These could assist in explaining, for instance, the behaviour of pharmacists who profess their support of new cognitive pharmacy services, but in practice, do not preferentially provide these services. ${ }^{28,29}$ Applying the participatory principle of 90 'nothing about us without us' ${ }^{\prime 8}$ into pharmacy practice, through research, could be transformational 91 for the community pharmacy setting. That said, participatory action research is not new to pharmacy practice. Various participatory action research methods have been used in studies, including the co-design and creation of pharmacy services, ${ }^{49,50}$ understanding how action research studies can change pharmacy work practices, ${ }^{51}$ and 
various hospital pharmacy improvements projects. ${ }^{52,53}$ This has included methods such as participatory and social mapping, pictures/photovoice, Spider-grams, ranking and scoring, seasonal calendars, life histories, narratives and storytelling, problem trees and human sculptures..$^{54}$ In general, different methods are used for exploration of subjective or social experiences, such as power hierarchies, social relationships, personal satisfaction with services, and understanding physical or social conditions. ${ }^{54}$

However, it has been reported that pharmacy practice research requires more experienced researchers in participatory action research and qualitative studies in general, ${ }^{51,54}$ and various factors can make its application complex. Participatory research requires a complex process that would be best guided by those with pre-existing experience. For example, the relationship between researcher and participants/collaborators is key, since trust is an important feature for the success of the study. Similarly, research teams must understand the research context well enough to both initiate and sustain the intervention in a way that is ethical and collaborative, requiring some flexibility of the team. The research process itself is sometimes multimodal and/or cyclical in nature, and may require constant negotiation with participants, plus ongoing reflection and reflexivity from the research team. However, the embedded nature of such research produces rich outcomes and a depth of meaning which can be transferrable to comparable settings. Its iterative nature means practice improvements and changes can result during the research. It is also particularly suited to constantly changing environments, such as the community pharmacy setting, due to the flexibility of the research process. ${ }^{51,54}$

VRE is one such participatory methodology that has become increasingly popular in the last decade, for studying (and intervening in) the complexity of frontline healthcare work. Drawing on ethnographic and participatory research traditions, researchers collaborate with study participants to record and select video footage of their everyday work practices. These videos are then replayed to the participants in reflexive discussions facilitated by researchers. Viewing the footage enables 
participants to view and analyse their actions as a third party, allowing them to distance themselves from their behaviour, thus making 'what is familiar strange' ${ }^{55}$ Aside from the video-recording of practices and the facilitation of reflexive discussions, VRE methods can also include observation, interviews, documentary analysis, and other ethnographic methods which allow the researcher to explore the context of interest. The primary aim of these methods is to make the complexity of their work practices more visible, and tangible, to practitioners, and hence encourage and inspire analysis and change. ${ }^{56}$

VRE has been used not only for research, but also as an intervention. Examples of previous interventions in healthcare settings worldwide include improving: infection control in hospitals, ${ }^{57,58}$ ICU clinical communication processes, ${ }^{59}$ inpatient dementia care, ${ }^{60}$ and shared decision making in primary care ${ }^{61}$ involving both clinicians and patients. In some cases, the methodology has been adopted by hospitals as their commitment to ongoing quality improvement, after successful completion of VRE projects. ${ }^{62}$ VRE represents an opportunity to review and improve clinical performance without the threat of deregistration, insurance claims or litigation, which can be rare for health professionals like pharmacists. ${ }^{63-65}$ VRE has also been used in pharmacist populations as part of two multidisciplinary studies. Kaiser Permanente Southern California South Bay Medical Center in the USA used VRE to improve their quality of transitional care by shadowing various assessments by health care professionals, including pharmacists ${ }^{66}$; and a current UK study protocol describes using VRE to investigate polypharmacy in $3 \mathrm{GP}$ clinics and 4 community pharmacies, including patient consultations from March 2016 to February $2021 .{ }^{67}$ Lastly, similar use of video in pharmacy practice education to enable students to reflect on and improve their counselling practices is well established. ${ }^{68}$

However, as a participatory action methodology, VRE does not just study recorded behaviours and actions for quality improvement. It also involves guided facilitation and questioning to elicit participant responses to: how the video has been captured by the researchers and/or participants; 
the social/physical/interactional contexts that are visible or not visible in the video; reflection on

reasoning for cinematographic choices and biographical context; and the contextualisation of

everyday care practices. This awareness of what is not visible in the footage allows the facilitator to

invite multiple meanings from various perspectives of those involved in the study, and provides

described below in greater detail.

To guide researchers new to the methodology, its theoretical framework has recently been articulated as a set of four guiding principles, exnovation, reflexivity, collaboration and $\operatorname{care}^{56}$ which are described as follows.

VRE acknowledges that the creative enactment of everyday clinical practice often goes unnoticed, perhaps due to repetition (habit) or the distraction and pace of busy workplaces. Those using VRE seek to make visible this 'local ecology of care' where the actions of a clinician can be seen as embedded in the workplace contexts where they unfold. This process of exnovation is designed to make tangible the complexity of healthcare work, and make visible the creativity, competence and expertise of healthcare workers.

In practice, this means that video-reflexive ethnographers are interested in how everyday practices unfold, creating (and making sense of) video footage of those practices in collaboration with frontline clinicians and patients.

For example, it has been established that community pharmacists commonly multi-task in order to complete their work. ${ }^{28,29,35,37,42}$ A VRE study might involve video recordings of the pharmacists working, and a reflexive discussion around how different tasks were prioritised and selected in the 
viewed footage. Responding to the footage might elicit comments about contextual pressures that change pharmacist task prioritisation (e.g. waiting customers), practitioner understanding of how the pharmacist role should be enacted (e.g. behaviours seen as acceptable/distasteful), or how pharmacist task priorities were shaped by professional socialisation (e.g. university/internship training and work experiences). This, in turn, could inform the researcher about how interplay of visible (through the video) and invisible (e.g. mental processes and preferences) factors might impact pharmacist negotiation of their tasks. This use of VRE could explore the subjective mental or cognitive workloads of pharmacists in depth, closely associated to their work contexts.

\section{Reflexivity}

Due to its incorporation into student curricula and professional development plans, ${ }^{70-72}$ pharmacists may be familiar with reflection as a personal activity for contemplating one's own actions, and an opportunity for learning. Reflexivity, however, as described in VRE, also takes into account the context in which actions and behaviours happen, such that participants' actions and behaviours are framed within the impact of others' actions and behaviours, and vice versa. ${ }^{69}$ In this way, reflexivity promotes critical awareness of individuals' conduct within layers of context, including their own backgrounds, socio-political context of their workplaces, physical environment, profession, and so on. ${ }^{73}$ For researchers, decisions of what should be video recorded and played back, and how reflexive sessions are held and facilitated are also determined through ongoing reflexivity. Reflexivity is expected from all parties involved (professionals, researchers and patients) and is necessary throughout all project stages. ${ }^{74}$

In practice, reflexive sessions involve researcher-facilitated viewings of recorded footage with participants, who may be those featured in the footage, colleagues, or others who are familiar with and/or have a stake in the practices shown. In viewing their footage, guided by researchers' questions, participants are invited to see their behaviour, within its context, from different viewpoints, and to respond to the complexity of the local healthcare setting. 
This enables a critical review of healthcare practices by stakeholders, encouraging a re-imagination of care: why do we work this way, is this appropriate, what else could be done, what could be improved? For example, in viewing footage, community pharmacists may become more aware of their multitasking as they describe their need for task prioritisation, and may see opportunities for prioritising their work differently. Researchers may find previous assumptions about pharmacist work and interaction with the work environment challenged at this stage. An example of researcher reflexivity will be described in the section below.

\section{Collaboration}

In line with the principle of exnovation, which seeks to foreground and harness frontline actors' expertise and understanding of their own practices, VRE invites stakeholders (e.g. pharmacists, dispensary technicians, patients) to participate as co-researchers, working alongside researchers to create and analyse footage, and to re-imagine and redesign work processes.

This collaboration is key, as stakeholders are active participants in the context of the health care setting, with an experiential understanding of its inner workings. Since researchers work with stakeholders to decide what footage is significant and should be recorded, videos discussed in a reflexive session are inherently important to both the researchers and stakeholders. The reflexive discussions arising from viewing the footage thus reflect researchers and stakeholders engaging in 'sense-making' of the practices under scrutiny. Findings of a VRE study are therefore produced through both 'insider' (i.e. stakeholders) and 'outsider' (i.e. researcher) perspectives, ${ }^{56,73}$ which may therefore be more readily translated into evidence-based, practical outcomes by combining theoretically-based researcher reflexivity, and experientially-based practitioner reflexivity. This strength of participatory research can be applied to many areas of pharmacy practice.

For example, within a community pharmacy, VRE researchers could consult with local pharmacists about what activities to record around cognitive pharmacy service provision. This could include pharmacist multitasking, patient recruitment, dispensary movements, use of counselling rooms, 

reflexive sessions, the researchers would then take on the role of a curious facilitator to make sense of footage with the pharmacists. This allows them to create meaning from the recordings together, rather than separately, at a distance from one another and the context at hand. This collaborative analysis of multitasking and task prioritisation in relation to cognitive pharmacy services could lead to a better understanding of pharmacists' behaviours when set in their context. These findings would lead to increased impact, since the context has already been taken into consideration and outcomes could be more practically implemented.

In this final principle, VRE researchers acknowledge that feelings of vulnerability are part of the VRE process, as the process of allowing one's behaviour to be recorded and examined by oneself and important others, including colleagues and managers, can be stressful and confronting. Video footage can also be easily misused, for instance, for performance monitoring by management who may impose sanctions, exposing vulnerability of participants with low control or power (e.g. patients and healthcare workers lower in the medical hierarchy). This is contrary to the purposes of VRE, which is designed to improve learning and interventions, rather than blame and personify errors. This last principle of care, therefore, emphasises the well-being of participants (and researchers) as a core tenet of the success of this methodology. The collaborative and reflexive processes of VRE enable researchers to monitor and negotiate situations and relationships with participants, to ensure that they are not adversely affected by participating in research. Furthermore, ensuring care for participants involves creating and maintaining their psychological safety, to enable open and frank discussion of recorded behaviours that are watched, edited and analysed by others. This is done through respectful engagement, facilitating open discussion, and an ongoing commitment to consent at every stage. For instance, this means that participants are regularly and continuously asked for their consent: to participate in the study, to be recorded, to have their footage kept and used, to have their footage shown to others, and so on. 
This is important in healthcare settings such as the community pharmacy sector, where organisational hierarchy and power structures can complicate pharmacists' autonomy and their subsequent practice. For example, in this era of patient-centred care, many pharmacists require professional autonomy to provide clinical care through cognitive pharmacy services. ${ }^{75,76}$ However, corporatisation of pharmacy business structures often result in the removal of some economic agency from the pharmacists accountable for the business, since their actions relate directly to pharmacy profitability. ${ }^{77}$ In one study, this was accompanied by a sense of loss of control and decision-making ability. ${ }^{76}$ In these situations, pharmacists may not have the economic autonomy to redirect resources towards increasing service quality due to the hierarchy of power in pharmacy organisations, particularly corporate and franchise business structures. ${ }^{77-79}$ Quite likely, this potential affront to professional autonomy may be related to the negative reactions of pharmacists towards service targets that focus on quantity. ${ }^{13,16,80}$ Thus, increased levels of strain reported in pharmacist populations may be directly related to power imbalances and conflict potentially unacknowledged by pharmacy leadership..$^{80-82}$

Much like the concept of a 'just culture' in patient safety, ${ }^{83}$ VRE creates a psychologically safe environment for clinicians to learn from observed footage and improve their care, and could be an important research approach to examine and potentially address participant work-related strain. process is customised to allow full and honest participation. A synergistic effect should be occurring where ideally, clinicians are the experts leading the researcher through their everyday care practices, which is in turn being facilitated and documented by the researcher-led processes for further analysis and learning. 
269 Above, we described how VRE enables participants and researchers to examine and intervene in the complexity of everyday work practices. In doing so, it may bridge the evidence-practice gap that characterises much of health services research and policy-making. ${ }^{84,85}$

In VRE, this is achieved by an interplay of closeness and distance: close engagement during research with practitioners and their actions embedded in their contexts; but also introducing 'distance', through the researcher's (outsider) presence and perspective; additionally, participants gain a kind of third-party perspective by watching their own footage. The effect of this 'distance' serves to direct practitioners' attention and interest to overlooked aspects of their practices, in order to reimagine them. Thus evidence is both created and applied, by practitioners and researchers, close to the relevant contexts of practice.

As a result of the COVID-19 pandemic, new challenges pertaining to distance have been introduced to VRE research, especially the physical distancing and travel restrictions for the prevention of community transmission. To adjust to this new problem of distance, in the next section an adaptation of the methods of VRE in one pilot study is discussed, through reflexive engagement with the four guiding principles.

\section{An adaptation for geographic restrictions and pandemic conditions}

As described in the examples above, cognitive service provision work in community pharmacies requires further investigation, since pharmacists appear not to provide these services despite demonstrating enthusiasm for them. ${ }^{13,15,16,86}$ Although community pharmacy practice internationally has transitioned towards a focus on cognitive pharmacy services, ${ }^{2,17,87-90}$ pharmacy productivity indicators remain predominantly tied to dispensing-related measures. ${ }^{41,91-93}$ This may have potentiated pharmacy worker strain and overload, ${ }^{81,94}$ by not taking into account the new ways of working involved in service provision. ${ }^{28,29} \mathrm{~A}$ mismatch between today's workload and its 
measurement are not easily investigated by traditional research methods (observations, surveys and

293

294

295

296

297

298

299

300

301

302

303

304

305

306

307 focus groups), which often take place 'at a distance' from the internal complexities of pharmacists' work practices..$^{35,37,41,42}$ It could be that pharmacist workload and productivity measures require updating in this new era of patient-centred care. In Australia, this could be because current workload and productivity measures tied to staffing and resource availability may be implicated in increased pharmacist workforce strain, causing dissatisfaction and turnover. ${ }^{28,29,81}$ This, in turn, may be complicated by multiple roles enacted by front-line pharmacists, ${ }^{95,96}$ some of which have arisen from research academics, professional associations and government policies. ${ }^{17}$

To explore how today's practice environment may affect the behaviour and actions of community pharmacists towards their work and cognitive pharmacy service provision, VRE was chosen for its collaborative, contextualised research approach.

Due to the remote nature of some Australian community pharmacies and the governmentmandated physical distancing during the COVID-19 pandemic, we designed a pilot study that took a participant-led approach to VRE, emphasising in particular the 'collaboration' guiding principle, and seeking to harness the expertise of community pharmacists as reflexive analysts of their own work practices.

With respect to the principle of care, we chose to request pharmacists to video record themselves and have them share and discuss this footage online with us, rather than potentially expose researchers, participants and communities to COVID-19. Instead of researchers physically being present to capture video footage onsite, participating pharmacists were asked to video-record short (10 minute) segments of their everyday work, and to submit this footage for use as research data, using their own devices. To protect the privacy of third parties such as patients and customers, any submitted video footage including their faces are de-identified using video-editing software. Participants are asked to not record any audio, and participants were also reminded to delete their own copies of footage involving third parties. As a further consideration, study participants are to 
inform and post study notices for pharmacy staff and patients, who are given the choice to view and delete any recordings they may be captured in.

In keeping with the principle of exnovation, researchers explained aims of the study and gave advice on selecting routine practices to participants to guide them in recording videos for submission. In the same way VRE researchers would normally have to justify what they record, participants were asked to reflexively consider their reasons for recording videos. At the point of footage submission, participants briefly described reasons for footage selection in writing. Video footage was then selected for use in the reflexive sessions based on participants' written rationales, research team review and study objectives.

In order to facilitate collaboration between researchers and participants, it was decided the reflexive sessions would be split into two stages. First, individual online sessions with a researcher would be held to elicit participant reflexivity. The second stage of reflexive sessions were designed for researchers to present the findings of the study to all participants who would be invited to attend, and garner their views on study outcomes. All reflexive sessions are audio and video recorded, and transcribed for content analysis. This two-stage design was chosen to allow sufficient care and reflexivity for researchers and participants, whilst maintaining an opportunity for multiple perspectives on study findings to be shared in a group setting.

This modification of the VRE process to include participant-led video recording is novel and enhances the participatory principles of VRE. By limiting researcher input into video recording, participants can take the lead in foregrounding their work practices, using their lived-in understanding of work contexts. Although support and guidelines for video recording were supplied, participants were told to consider what they felt important about pharmacist work when choosing what to record and submit. The research team was then 'introduced' by participants to these contextualised insights in reflexive sessions for a truly collaborative participant-led approach. This requires participants to engage in reflexivity regarding their selection of footage throughout the process. 
Further, the research team consists of a currently practising community pharmacist completing doctoral studies $(*)$, a pharmacist professor $\left({ }^{* *}\right)$ and a health services researcher and social scientist $\left({ }^{* *}\right)$. During the planning of this pilot study, numerous discussions surrounding researcher roles during the reflexive sessions were held. It was decided that $\left({ }^{*}\right)$, with an insider view into this work context, would conduct these sessions to assist in drawing out implied and assumed practitioner knowledge. Specific care was also taken to consider what care and collaboration signified in this study. For example, pharmacists are accustomed to tailoring their social interactions to their role partners. ${ }^{75}$ It was thus important to continually reinforce the research team's desire for participants to demonstrate their expertise of their practice, rather than catering to perceived researcher desires. For instance, during reflexive sessions, an active listening approach was used to reflect participant statements back to them, and confirm views to avoid assumptions.

This participant-led approach contrasts with the previously established role of the researcher in carrying out VRE. Typically, video-reflexive ethnographers negotiate video footage recording with front-line clinicians and patients, making methodological decisions surrounding camera point-ofviews and similar considerations. However, rather than being a disadvantage, this adaptation remains true to the VRE principles of exnovation, reflexivity, collaboration, and care.

Ethnography is, in itself, a process in which the researcher can contextualise the research data into the lives of the participant in question. This requires time and understanding through the use of observations, note-taking and reflexive commenting from both the researcher and the participant in VRE. We consider that this particular use of VRE was offering a form of cultural brokerage ${ }^{97}$ to pharmacists who complained their work was not recognised in an earlier unpublished study by $(*)$, some of whom also opted-in to this study. It may have been empowering for pharmacists who may feel their professionalism is unseen, and their concerns for their occupational health unheard. It could be that community pharmacists, in wanting to appear as professional healthcare clinicians, 
have been rather too successful in performing their work in an easy, streamlined manner. When designing this study, the intention had been to describe the complex world of the community pharmacist and the rich interactional work they participate in, including the unseen restrictions represented by legal, professional and ethical frameworks. Being conscious of the positivist traditions in the profession, this methodology was chosen to peel back the layers of factors that motivate pharmacists to behave as they do. Treating the video footage as visual records of their work practices, $\left({ }^{*}\right)$ asked questions about their reasons for capturing their footage, and their rationale for their particular way of working, thus requiring them to view their own setting, practices and professional identity with reflexivity. Although viewing of video footage alone was not ethnographic, the reflexive discussions it sparked allowed pharmacists to assign unseen meaning to particular processes. For example, one participant rejected the idea that a community pharmacist was merely a medication expert or accessible health professional, instead strongly advocating that all pharmacist tasks were representative of different parts of their professional identity, which needed to be described by multiple terms. The description of their working process revolved around their workflow, patient needs and time afforded to them between patient interactions, wherein they slotted in administrative tasks such as updating restricted drug records and follow-up patient calls. Challenges of VRE adaptation within the COVID-19 pandemic in the Australian setting

As a pharmacist-researcher $\left({ }^{*}\right)$, the differing paradigms of pharmacy practice research and community practice have become clearer. Although pharmacist clinicians typically write from a positivist paradigm (as other health professionals do, and struggle to depart from), ${ }^{98}$ it could be the ability of community pharmacists to contextualise advice and appreciate multiple client realities ${ }^{75}$ demonstrates appropriateness in utilising this participatory approach. A constructivist approach compatible with symbolism and sense-making is required for both negotiating the complexities of everyday healthcare practices, and engaging in research co-analysis with participants. Rather than imposing the researcher's own perspectives upon observations and participant dialogue, VRE enables the researcher to work with stakeholders to both create and interpret findings. As such, VRE 
co-analysis and flexibility towards multiple meanings necessitates a critical research framing. This

394

395

396

397

398

399

400

401

402

403

404

405

406

407

408

409

410

411

412

413

414

415

416

417

may have some challenges for clinicians and researchers who are unfamiliar with the social sciences. ${ }^{98}$

However, the acceptability of this study was not high during this pandemic: the length of involvement and enthusiasm required from participants led to recruitment challenges, particularly as workloads were already increased by COVID-19 regulations (as explained by our participants). Although there was some initial interest when we began recruitment, many did not respond beyond their expressions of interest. One pharmacist cited a lack of approval from their proprietor who had read the disclaimer for disclosure of illegal behaviour, despite the fact their employee would be selfrecording and selecting footage involved. Another described with great distress how they were overworked and could not participate, due to concurrent participation in a different study. Overall, it appeared that participants with previous involvement with research or interaction with the research team were more likely to participate. Additionally, those who believed pharmacists could be better acknowledged for their work were more motivated to produce better quality data. Whilst engagement with pharmacists is originally described to be difficult, it may be even harder to recruit for a VRE study from a distance, where the relationship between researcher and participants is key. It was with some irony we noted we were asking pharmacists to do additional 'work' for the study in order to understand their initial workload problems.

\section{Implications}

Although the pilot study described in this paper did not have the scope to include pharmacy staff, patients and collaborative health professionals, findings from this study could influence a larger scale study design that would be more approachable for pharmacists, and thus amenable to the inclusion of other parties.

Furthermore, participatory research approaches like VRE could encourage research engagement in community pharmacists, since they have an opportunity to direct research towards issues of interest 
towards themselves, and see their potential benefit first-hand. One suitable topic, as described above, might be about pharmacist interactional and mental work processes. ${ }^{31,35,37,42}$ Workforce research would also benefit from the use of VRE, since it would situate workforce issues within workplace contexts, including unseen stresses/pressure and role conflicts. As learning interventions for the implementation of services, and cognitive pharmacy service quality improvement, the use of VRE could be investigated further, since associated pharmacist behaviour and actions are not well documented. Other areas that are traditionally difficult to investigate could potentially be addressed through VRE, such as how Aboriginal and Torres Strait Islander communities interact with pharmacy services; how persons of migrant, refugee and disadvantaged backgrounds experience pharmacies; and the use of translator services. Drawing on the success of VRE in investigating these issues with clinicians in hospitals, other areas of application could include the use of protocols with pharmacy support staff (i.e. pharmacy assistants and technicians), information handovers, and professional identity. In general, VRE could be an useful and flexible method, due to its caring, participatory and collaborative nature, and sensitivity towards participants.

VRE can be effective in pharmacy settings as both an intervention and an exploratory research approach. A key characteristic of VRE is the flexibility afforded to researchers in conducting research, in return for constant reflexivity about methodological principles, as demonstrated above. For researchers, a thorough understanding of the theoretical underpinnings of VRE are therefore important to ensure appropriate use..$^{24,56}$

VRE presents an exciting opportunity for pharmacy practice researchers to solve questions such as the contrary behaviour of front-line clinicians who profess approval of cognitive pharmacy services but do not always provide them. Furthermore, it gives voice to front-line community pharmacists, and potentially in the future, pharmacy staff and patients. This may encourage research engagement and future scholarship. This could be an effective way to explore issues in the pharmacist workforce 
undergoing role transition. ${ }^{99}$-As an established methodology, VRE can engage with complexity and

contradictions in human behaviour, which may be highly transformative in future pharmacy practice

1. Baldwin JN, Bootman JL, Carter RA, et al. Pharmacy Practice, Education, and Research in the

2. Moles RJ, Stehlik P. Pharmacy Practice in Australia. The Canadian journal of hospital pharmacy. 2015;68:418-426.

3. Malet-Larrea A, García-Cárdenas V, Sáez-Benito L, Benrimoj SI, Calvo B, Goyenechea E. Costeffectiveness of professional pharmacy services in community pharmacy: a systematic review. Expert Rev Pharmacoecon Outcomes Res. 2016;16:747-758.

4. Gammie T, Vogler S, Babar Z-U-D. Economic Evaluation of Hospital and Community Pharmacy Services. Ann Pharmacother. 2016;51:54-65.

5. Publications Committee of the American College of Clinical P, Schumock GT, Meek PD, Ploetz PA, Vermeulen LC. Economic Evaluations of Clinical Pharmacy Services-1988-1995. Pharmacotherapy: The Journal of Human Pharmacology and Drug Therapy. 1996;16:11881208.

6. Touchette DR, Doloresco F, Suda KJ, et al. Economic evaluations of clinical pharmacy services: 2006-2010. Pharmacotherapy. 2014;34:771-793.

7. Benrimoj SI, Langford JH, Berry G, et al. Economic Impact of Increased Clinical Intervention Rates in Community Pharmacy. Pharmacoeconomics. 2000;18:459-468.

8. Schumock GT, Butler MG, Meek PD, et al. Evidence of the Economic Benefit of Clinical Pharmacy Services: 1996-2000. Pharmacotherapy: The Journal of Human Pharmacology and Drug Therapy. 2003;23:113-132.

9. Pharmacists in 2023: For patients, for our profession, for Australia's health system. Canberra: The Pharmaceutical Society of Australia Ltd. ; 2019.

10. Cutler RL. The UTS Community Pharmacy Barometer. Sydney, Australia: University of Technology Sydney; 2018.

11. B Saini, M Brillant, J Filipovska, et al. Recruitment and retention of community pharmacists in pharmacy practice research. In: Saini B, ed. Sydney, Australia: Faculty of Pharmacy, University of Sydney; 2005.

12. Moullin JC, Sabater-Hernández D, Fernandez-Llimos F, Benrimoj SI. Defining professional pharmacy services in community pharmacy. Research in Social and Administrative Pharmacy. 2013;9:989-995.

13. Bond CM, Blenkinsopp A, Inch J, Celino G, Gray NJ. The effect of the new community pharmacy contract on the community pharmacy workforce. Medicines \& People: Turning knowledge into Know-How: The Pharmacy Practice Research Trust; 2008.

14. Gidman WK, Hassell K, Day J, Payne K. Does community pharmacy offer women familyfriendly working conditions and equal opportunities? the accounts of female community pharmacists over the age of 30. Int J Pharm Pract. 2007;15:53-59.

15. Gidman WK, Hassell K, Day J, Payne K. The impact of increasing workloads and role expansion on female community pharmacists in the United Kingdom. Res Social Adm Pharm. 2007;3:285-302.

16. Gidman W. Increasing community pharmacy workloads in England: causes and consequences. Int J Clin Pharm. 2011;33:512-520.

17. Kellar J, Paradis E, van der Vleuten CPM, oude Egbrink MGA, Austin Z. A Historical Discourse Analysis of Pharmacist Identity in Pharmacy Education. Am J Pharm Educ. 2020:ajpe7864. 
18. Kuipers $E$, Wensing $M$, De Smet PAGM, Teichert M. Barriers and facilitators for community pharmacists' participation in pharmacy practice research: a survey. The International journal of pharmacy practice. 2019;27:399-402.

19. Awaisu A, Alsalimy N. Pharmacists' involvement in and attitudes toward pharmacy practice research: A systematic review of the literature. Res Social Adm Pharm. 2015;11:725-748.

20. Crilly P, Patel N, Ogunrinde A, Berko D, Kayyali R. Community Pharmacists' Involvement in Research in the United Kingdom. Pharmacy. 2017;5:48.

21. Perreault MM, Thiboutot Z, Burry LD, et al. Canadian Survey of Critical Care Pharmacists' Views and Involvement in Clinical Research. Ann Pharmacother. 2012;46:1167-1173.

22. Bhagavathula AS, Gebreyohannes EA, Gebresillassie BM, Erku DA, Negesse CT, Belay YB. Community pharmacists' interest in and attitude to pharmacy practice research in Ethiopia: A cross-sectional study. PLoS One. 2017;12:e0178919.

23. Lee R, Dahri K, Lau TTY, Shalansky S. Perceptions of Hospital Pharmacists Concerning Clinical Research: A Survey Study. The Canadian journal of hospital pharmacy. 2018;71:105-110.

24. Hadi MA, José Closs S. Ensuring rigour and trustworthiness of qualitative research in clinical pharmacy. Int J Clin Pharm. 2016;38:641-646.

25. Winit-Watjana W. Research philosophy in pharmacy practice: necessity and relevance. Int J Pharm Pract. 2016;24:428-436.

26. Denzin NK, Lincoln YS. The SAGE handbook of qualitative research. Thousand Oaks: Sage; 2011.

27. Rybakov KN, Beckett R, Dilley I, Sheehan AH. Reporting quality of survey research articles published in the pharmacy literature. Res Social Adm Pharm. 2020.

28. Yong FR, Garcia-Cardenas V, Williams KA, Benrimoj SI. Factors affecting community pharmacist work: a scoping review and thematic synthesis using role theory. Res Social Adm Pharm. 2020;16:123-141.

29. Yong FR. Instruments measuring community pharmacist role stress and strain measures: A systematic review. Research in Social and Administrative Pharmacy. 2020.

30. Lea VM, Corlett SA, Rodgers RM. Describing interruptions, multi-tasking and task-switching in community pharmacy: a qualitative study in England. Int J Clin Pharm. 2015;37:1086-1094.

31. Chui MA, Stone JA. Exploring information chaos in community pharmacy handoffs. Res Social Adm Pharm. 2014;10:195-203.

32. Thompson L, Bidwell S. Space, time, and emotion in the community pharmacy. Health \& Place. 2015;34:251-256.

33. Rapport F, Doel MA, Jerzembek GS. "Convenient space" or "a tight squeeze": insider views on the community pharmacy. Health Place. 2009;15:315-322.

34. Family HE, Weiss M, Sutton J. The effects of mental workload on community pharmacists' ability to detect dispensing errors. Pharm Res UK. 2013:1-121.

35. Chui MA, Look KA, Mott DA. The association of subjective workload dimensions on quality of care and pharmacist quality of work life. Res Social Adm Pharm. 2014;10:328-340.

36. Gaither CA, Kahaleh AA, Doucette WR, Mott DA, Pederson CA, Schommer JC. A modified model of pharmacists' job stress: the role of organizational, extra-role, and individual factors on work-related outcomes. Res Soc Adm Pharm. 2008;4:231-243.

37. Chui MA, Mott DA. Community pharmacists' subjective workload and perceived task performance: a human factors approach. J Am Pharm Assoc (2003). 2012;52:e153-e160.

38. Schommer JC, Pedersen CA, Doucette WR, Gaither CA, Mott DA. Community pharmacists' work activities in the United States during 2000. J Am Pharm Assoc (Wash). 2002;42:399406.

39. Benrimoj SI, Frommer MS. Community pharmacy in Australia. Aust Health Rev. 2004;28:238246.

40. Grasha AF, Schell K. Psychosocial factors, workload, and human error in a simulated pharmacy dispensing task. Percept Mot Skills. 2001;92:53-71. 
41. Hassell K, Seston EM, Schafheutle El, Wagner A, Eden M. Workload in community pharmacies in the UK and its impact on patient safety and pharmacists' well-being: A review of the evidence. Health and Social Care in the Community. 2011;19:561-575.

42. Chui MA, Mott DA, Maxwell L. A qualitative assessment of a community pharmacy cognitive pharmaceutical services program, using a work system approach. Res Social Adm Pharm. 2012;8:206-216.

43. Rieck $A$, Pettigrew $S$. How physician and community pharmacist perceptions of the community pharmacist role in Australian primary care influence the quality of collaborative chronic disease management. Quality in primary care. 2013;21:105-111.

44. Schommer JC, Gaither CA. A segmentation analysis for pharmacists' and patients' views of pharmacists' roles. Res Social Adm Pharm. 2014;10:508-528.

45. van Eikenhorst L, Salema NE, Anderson C. A systematic review in select countries of the role of the pharmacist in consultations and sales of non-prescription medicines in community pharmacy. Research in Social and Administrative Pharmacy. 2017;13:17-38.

46. Gidman W, Cowley J. A qualitative exploration of opinions on the community pharmacists' role amongst the general public in Scotland. Int J Pharm Pract. 2013;21:288-296.

47. Khan MU, Khan AN, Ahmed FR, et al. Patients' opinion of pharmacists and their roles in health care system in Pakistan. J Young Pharm. 2013;5:90-94.

48. Bridges D. 'Nothing About Us Without Us': The Ethics of Outsider Research2017:341-361.

49. Elliott RA, Lee $\mathrm{CY}$, Beanland $\mathrm{C}$, et al. Development of a clinical pharmacy model within an Australian home nursing service using co-creation and participatory action research: the \&lt;em\&gt;Vi\&lt;/em\&gt;siting \&lt;em\&gt;P\&lt;/em\&gt;harmacist (ViP) study. BMJ Open. 2017;7:e018722.

50. Dineen-Griffin S, Benrimoj SI, Williams KA, Garcia-Cardenas V. Co-design and feasibility of a pharmacist-led minor ailment service. BMC Health Serv Res. 2021;21:80.

51. Nørgaard LS, Sørensen EW. Action research methodology in clinical pharmacy: how to involve and change. Int J Clin Pharm. 2016;38:739-745.

52. van Buul LW, Sikkens JJ, van Agtmael MA, Kramer MH, van der Steen JT, Hertogh CM. Participatory action research in antimicrobial stewardship: a novel approach to improving antimicrobial prescribing in hospitals and long-term care facilities. J Antimicrob Chemother. 2014;69:1734-1741.

53. Loewenson R, Flores W, Shukla A, et al. Raising the profile of participatory action research at the 2010 Global Symposium on Health Systems Research. MEDICC Rev. 2011;13:35-38.

54. Bradley H. Participatory action research in pharmacy practice. Pharmacy practice research methods: Springer; 2015:91-105.

55. Mannay D. Visual, Narrative and Creative Research Methods: Application, Reflection and Ethics2015.

56. ledema R, Carroll K, Collier A, Hor S-y, Mesman J, Wyer M. Video-Reflexive Ethnography in Health Research and Healthcare Improvement: Theory and Application. Florida, USA: CRC Press, Taylor \& Francis Group, LLC; 2019.

57. ledema $\mathrm{R}$, Hor $\mathrm{S}$, Wyer $\mathrm{M}$, et al. An innovative approach to strengthening health professionals' infection control and limiting hospital-acquired infection: video-reflexive ethnography: BMJ; 2015.

58. Gilbert GL, Hor S, Wyer M, Sadsad R, Badcock C-A, ledema R. Sustained fall in inpatient MRSA prevalence after a video-reflexive ethnography project; an observational study. Infection, Disease \& Health. 2020;25:140-150.

59. Carroll K, ledema R, Kerridge R. Reshaping ICU Ward Round Practices Using Video-Reflexive Ethnography. Qual Health Res. 2008;18:380-390.

60. Hung L, Phinney A, Chaudhury H, Rodney P. Using Video-Reflexive Ethnography to Engage Hospital Staff to Improve Dementia Care. Global Qualitative Nursing Research. 2018;5:2333393618785095. 
61. Mcleod HM. Respect and shared decision making in the clinical encounter, A Video-Reflexive Ethnography [Dissertation]. Minnesota, USA: Health Services Research, Policy and Administration, University of Minnesota; 2017.

62. Carroll K, Mesman J. Multiple Researcher Roles in Video-Reflexive Ethnography. Qual Health Res. 2018;28:1145-1156.

63. Lee YC, Wu HH, Hsieh WL, Weng SJ, Hsieh LP, Huang CH. Applying importance-performance analysis to patient safety culture. Int J Health Care Qual Assur. 2015;28:826-840.

64. Nordén-Hägg A, Kälvemark-Sporrong S, Lindblad AK. Exploring the relationship between safety culture and reported dispensing errors in a large sample of Swedish community pharmacies. BMC Pharmacology and Toxicology. 2012;13.

65. Johnson SJ, O'Connor EM, Jacobs S, Hassell K, Ashcroft DM. The relationships among work stress, strain and self-reported errors in UK community pharmacy. Res Social Adm Pharm. 2014; 10:885-895.

66. Neuwirth EB, Bellows J, Jackson AH, Price PM. How Kaiser Permanente Uses Video Ethnography Of Patients For Quality Improvement, Such As In Shaping Better Care Transitions. Health Aff (Millwood). 2012;31:1244-1250.

67. Swinglehurst $D$, Fudge N. Addressing the polypharmacy challenge in older people with multimorbidity (APOLLO-MM): study protocol for an in-depth ethnographic case study in primary care. BMJ Open. 2019;9:e031601.

68. Jin HK, Choi JH, Kang JE, Rhie SJ. The effect of communication skills training on patientpharmacist communication in pharmacy education: a meta-analysis. Advances in Health Sciences Education. 2018;23:633-652.

69. ledema R, Mesman J, Carroll K. Visualising Health Care Practice Improvement: Innovation from Within2013.

70. Fragkos KC. Reflective practice in healthcare education: an umbrella review. Education Sciences. 2016;6:27.

71. Austin Z, Marini A, Desroches B. Use of a learning portfolio for continuous professional development: A study of pharmacists in Ontario (Canada). Pharmacy Education. 2005;5.

72. Micallef R, Kayyali R. A systematic review of models used and preferences for continuing education and continuing professional development of pharmacists. Pharmacy. 2019;7:154.

73. McHugh SK, Lawton R, Hara JK, Sheard L. Does team reflexivity impact teamwork and communication in interprofessional hospital-based healthcare teams? A systematic review and narrative synthesis. BMJ Quality \&amp;amp; Safety. 2020;29:672.

74. Collier A, Wyer M. Researching Reflexively With Patients and Families: Two Studies Using Video-Reflexive Ethnography to Collaborate With Patients and Families in Patient Safety Research. Qual Health Res. 2015;26:979-993.

75. Duckett K. Community, autonomy and bespoke services: Independent community pharmacy practice in hyperdiverse, London communities. Research in Social and Administrative Pharmacy. 2015;11:531-544.

76. Dobson RT, Perepelkin J. Pharmacy ownership in Canada: implications for the authority and autonomy of community pharmacy managers. Res Social Adm Pharm. 2011;7:347-358.

77. Bush J, Langley CA, Wilson KA. The corporatization of community pharmacy: implications for service provision, the public health function, and pharmacy's claims to professional status in the United Kingdom. Res Soc Adm Pharm. 2009;5:305-318.

78. Kahaleh A, Gaither CA. Effects of empowerment on pharmacists' organizational behaviors. J Am Pharm Assoc (2003). 2005;45:700-708.

79. Kahaleh A, Gaither $C$. The effects of work setting on pharmacists' empowerment and organizational behaviors. Res Social Adm Pharm. 2007;3:199-222.

80. Eden $\mathrm{M}$, Schafheutle El, Hassell K. Workload pressure among recently qualified pharmacists: an exploratory study of intentions to leave the profession. Int J Pharm Pract. 2009;17:181187. 
81. Jacobs S, Hassell K, Ashcroft D, Johnson S, O'Connor E. Workplace stress in community pharmacies in England: associations with individual, organizational and job characteristics. J Health Serv Res Policy. 2014;19:27-33.

82. Perepelkin J, Dobson RT. Influence of ownership type on role orientation, role affinity, and role conflict among community pharmacy managers and owners in Canada. Res Social Adm Pharm. 2010;6:280-292.

83. Norden-Hagg A, Sexton JB, Kalvemark-Sporrong S, Ring L, Kettis-Lindblad A. Assessing safety culture in pharmacies: the psychometric validation of the Safety Attitudes Questionnaire (SAQ) in a national sample of community pharmacies in Sweden. BMC Clin Pharmacol. 2010;10:8.

84. Dopson S, Locock L, Gabbay J, Ferlie E, Fitzgerald L. Evidence-Based Medicine and the Implementation Gap. Health (N Y). 2003;7:311-330.

85. Lau R, Stevenson $F$, Ong BN, et al. Achieving change in primary care-causes of the evidence to practice gap: systematic reviews of reviews. Implementation Science. 2016;11:40.

86. Luetsch $\mathrm{K}$. Attitudes and attributes of pharmacists in relation to practice change $-\mathrm{A}$ scoping review and discussion. Res Social Adm Pharm. 2017;13:440-455.e411.

87. Campbell C, Braund R, Morris C. Beyond the four walls: an exploratory survey of location, employment and roles of pharmacists in primary health care. J Prim Health Care. 2017;9:297-310.

88. Bernsten C, Andersson K, Gariepy $\mathrm{Y}$, Simoens S. A comparative analysis of remuneration models for pharmaceutical professional services. Health Policy. 2010;95:1-9.

89. Knoer SJ, Eck AR, Lucas AJ. A review of American pharmacy: education, training, technology, and practice. Journal of Pharmaceutical Health Care and Sciences. 2016;2:32.

90. Sporrong SK, Hoglund AT, Hansson MG, Westerholm P, Arnetz B. "We are white coats whirling round"--moral distress in Swedish pharmacies. Pharm World Sci. 2005;27:223-229.

91. Jacobs $S$, Bradley F, Elvey R, et al. Investigating the organisational factors associated with variation in clinical productivity in community pharmacies: a mixed-methods study 2017.

92. Lea VM, Corlett SA, Rodgers RM. Workload and its impact on community pharmacists' job satisfaction and stress: a review of the literature. Int J Pharm Pract. 2012;20:259-271.

93. Rutter PM, Brown D, Jones IF. Pharmacy research: the place of work measurement. Int J Pharm Pract. 1998;6:46-58.

94. Chapman CB, Wilson SG, Wilson DI, Dunkley MK. National survey of pharmacists, intern pharmacists and pharmacy students in Australia: the nature and extent of stress and wellbeing. Int J Pharm Pract. 2020;n/a.

95. Schindel TJ, Yuksel N, Breault R, Daniels J, Varnhagen S, Hughes CA. Perceptions of pharmacists' roles in the era of expanding scopes of practice. Research in social \& administrative pharmacy : RSAP. 2017;13:148-161.

96. Elvey R, Hassell K, Hall J. Who do you think you are? pharmacists' perceptions of their professional identity. Int J Pharm Pract. 2013;21:322-332.

97. Pink S. Doing Visual Ethnography. Second Edition ed. London: SAGE Publications, Ltd; 2007.

98. Rathbone AP, Jamie K. Transferring from Clinical Pharmacy Practice to Qualitative Research: Questioning Identity, Epistemology and Ethical Frameworks. Sociological Research Online. 2016;21:1-9.

99. Latour B. Why Has Critique Run out of Steam? From Matters of Fact to Matters of Concern. Critical Inquiry. 2004;30:225-248. 